\title{
Addressing the Disparity in Refugee Mental Health Services: a Pilot Study of a Traumatic Stress Intervention Utilizing a Language-Free mHealth Application
}

*For correspondence: emily.mazzulla@marquette.edu (EM)

\section{Emily C. Mazzulla1*, Karen M. Fondacaro², Holly Weldon ${ }^{3}$, Marguerite Dibble $^{4}$, Matthew Price ${ }^{3}$}

${ }^{1}$ Marquette University, Milwaukee, WI, USA; ${ }^{2}$ New England Survivors of Torture and Trauma (NESTT), Burlington, VT, USA; ${ }^{3}$ University of Vermont, Burlington, VT, USA;

${ }^{4}$ GameTheory, Burlington, VT, USA; ${ }^{3}$ University of Vermont, Burlington, VT, USA

Abstract Objective: After resettlement, an overwhelming number of refugees struggle with Chronic Traumatic Stress (CTS), the persistence of traumatic events (e.g., re-experiencing past trauma; news of on-going war) coupled with daily post-migration stressors (e.g., poverty, lack of transportation). CTS significantly increases the burden of mental health challenges experienced by refugees. Evidence-based mental health treatments often rely on worksheets, mobile applications, websites, or telephone calls to facilitate the management of distress outside of treatment sessions. Language barriers prevent these strategies from being incorporated into mental health treatment for refugees, which results in a significant disparity in care. Treatments delivered via mobile devices can address this barrier through the use of intuitive images that eliminate the need for text or language-based instruction.

Methods: A six-week pilot study assessing the effectiveness of group intervention utilizing a language free, culturally relevant mobile health (mHealth) application was conducted in a sample of Somali-Bantu and Nepali-Bhutanese adult refugee men and women $(\mathrm{N}=18)$. Paired-samples t-tests were conducted to compare pre- and post-intervention levels of psychosocial distress, anxiety, depression, and traumatic stress, on the Refugee Health Screener (RHS-15) and an investigator generated coping measure.

Results: Results indicated significant reduction ( $p<.001)$ in symptoms related to traumatic stress, anxiety, depression and somatic complaints in addition to a significant increase $(p<.001)$ in the use of coping skills.

Conclusions: The use of a mobile mental health app, in combination with in-person therapy, was effective in reducing mental health symptomology and in increasing the use of coping skills in Somali-Bantu and Nepali-Bhutanese refugees. 


\section{Proper Citation and Use of Work}

This work has been published in the the Journal of Technology in Behavioral Science with the doi: https://doi.org/10.1007/s41347-021-00213-7

When referencing this work, please the following citation:

Mazzulla, E. C., Fondacaro, K. M., Weldon, H., Dibble, M., Price, M. (2021). Addressing the Disparity in Refugee Mental Health Services: a Pilot Study of a Traumatic Stress Intervention Utilizing a Language-Free mHealth Application. Journal of Technology in Behavioral Science, 1-10.

\section{Introduction}

The United States is home to over 500,000 refugees who are survivors of trauma, torture, and war atrocities (Campbell, 2007). Coupled with experiencing post-trauma symptoms, refugees often are exposed to additional traumatic events (e.g., family affected by ongoing war) and postmigration stressors (e.g., poverty, employment problems, lack of transportation) (Fondacaro Mazzulla, 2018). The nature of traumatic stress results in an undue burden of mental illness characterized by a markedly high prevalence of posttraumatic stress disorder (PTSD; 69-92\%) Uohnson Thompson, 2008; Moisander Edston, 2003), depression, generalized anxiety disorder, and somatic concerns (Jaranson et al., 2004; Mollica et al., 1997). Not surprisingly, functional impairment ensues, requiring culturally adapted assessment and treatment. Evidence-based post-traumatic stress, anxiety, and depression interventions focus on exposure-based techniques, symptom reduction, valued living, emotion regulation, and coping skills to manage acute distress (Nicholl Thompson, 2004). These interventions are grounded in cognitive-behavioral (CBT) and acceptance and commitment therapy frameworks (ACT). A primary challenge of treatment provision is the management of symptoms between sessions and maintenance of treatment gains after treatment is completed (Glenn et al., 2013). Whereas clinicians working with English fluent populations often use telephone communication (Rose et al., 2015; Stecker et al., 2014), worksheets (Foa et al., 2008; Resick Schnicke, 1993), or websites (Amstadter et al., 2009), multiple barriers prevent the effective use of these strategies with refugees. Many re-settled refugees have limited English proficiency, making the use of written materials (e.g., handouts or websites) challenging, thereby limiting the number of tools available for facilitating between session intervention. Transportation is often limited such that patients are unable to travel to providers in times of crisis. Clinicians are left to rely exclusively on the patient, who may be in extreme distress, to be able to utilize skills recalled from a therapeutic session. This results in a marked disparity in the quality of care for refugees who are English language learners. As a result, refugees use high-cost services, such as hospital emergency rooms, in response to acute distress (Fernandez et al., 2004; Miller, 1995). Language-free treatments to facilitate the use of skills between sessions and after intervention are greatly needed to reduce the disparity in care.

Mobile technology, or mHealth, treatments are a means to address this disparity (Price et al., 2014). Although many applications have been developed for English speaking patients receiving treatment (Kuhn et al., 2014; Luxton et al., 2011), they are poorly suited for refugees, and most are not evidence-based. Existing applications rely on English text to communicate with the user. Many applications for trauma symptoms were developed for combat veterans and contain military images (Bush et al., 2013; Luxton et al., 2011; Ruzek et al., 2011), which can be inappropriate for refugees who are victims of war atrocities. Furthermore, no developed applications on the market to date are designed specifically for refugees originating from non-western cultures. Cultural adaptations to interventions and resources are imperative for this population as they help to establish trust and promote compliance (Fondacaro Harder, 2014). Thus, there is a need for 
$\mathrm{mHealth}$ interventions specifically designed for refugees to close the current gap in refugee mental health care.

Importantly, prior literature reveals that mobile device ownership and interest in applications for healthcare among diverse populations, is high (Price et al., 2013). Moreover, the vast majority (82\%) of adults in the United States own app-enabled mobile devices (Krebs Duncan, 2015) and average smart phone users check their devices as often as 150 times per day (Bakker et al., 2016). According to a relevant study, $65.5 \%$ of respondents who downloaded a health app said they opened the app at least once per day and used it for approximately 1-10 minutes per day (Krebs Duncan, 2015). When examining usage of mental health apps, a public survey found that $76 \%$ of respondents would be interested in using smartphones for monitoring mental health (Bakker et al., 2016). However, experimental validation of mobile mental health apps is seriously lacking and most health apps have not been designed with input from healthcare professionals (Krebs Duncan, 2015), or specifically from the refugee population. There is clearly a need for evidence-based mobile mental health applications that are designed for refugees with mental health concerns.

NESTT, an application developed by the authors, based on the Chronic Traumatic Stress Framework (CTS) (Fondacaro Mazzulla, 2018) and CTS-Treatment (CTS-T) (Mazzulla Fondacaro, 2018), was designed specifically for refugee clients presenting with symptoms associated with posttraumatic stress disorder, anxiety, and depression. The Chronic Traumatic Stress Framework and affiliated treatment was developed specially for resettled refugees and is a non-pathologizing, culturally responsive treatment approach to increase coping skills and address symptoms related to trauma and post-migration living difficulties. The application utilizes evidence-based cognitive behavioral and acceptance-based therapy techniques, and encourages the reporting of thoughts, feelings and behaviors with real-time engagement. For example, subjective levels of experienced emotion are assessed before, during, and after use of application components. Additionally, behavioral activation such as activity planning, and coping techniques such as deep breathing and progressive muscle relaxation, are included in the application. Finally, the language free, simple interface is uniquely designed with extensive contribution from cultural consultants for refugees from Africa (e.g., Somali-Bantu) and Asia (e.g., Nepali-Bhutanese) (Bakker et al., 2016). The primary aim of this study was to conduct a pilot intervention utilizing the NESTT application. We expected that patient engagement, a critical component of technology-based interventions (Kiluk et al., 2017), would be high. Moreover, we expected that there would be a reduction from pre to post intervention in symptoms associated with anxiety, depression, somatization, and post-traumatic stress, and an increase in coping skills and valued living.

\section{Methods}

\section{Participants}

Twenty adult participants were recruited from Somali Bantu and Nepali-Bhutanese communities re-settled in a small new England city. After the first week, one male participant chose to discontinue participation and one male participant needed to postpone participation, resulting in a final sample of $\mathrm{N}=18$ participants. A six-week pilot test was conducted in a group format (5 Somali-Bantu Women/5 Nepali-Bhutanese Women-group 1; 3 Somali-Bantu Men/5 NepaliBhutanese Men-group 2) during which the NESTT application was utilized.

\section{Development of NESTT Application}

The NESTT app was developed by a software development team, mental health experts, and cultural consultants. Whereas prior applications rely on English text instructions (Kuhn et al., 
2014), usually written at an eighth-grade reading level, the application used in the present study is language-free, without any text, and incorporates culturally validated visual cues to guide users through specific therapeutic techniques. The NESTT app was developed with extensive community engagement through a Community Based Participatory Research (CBPR) (Minkler Wallerstein, 2011) design. Specifically, a panel of community advisors was established including interpreters, refugee community leaders, and experienced clinicians. NESTT was developed using an agile development process by incorporating frequent feedback across multiple iterations of coding to improve the application. Each tool included in the application was designed without languagebased instruction and incorporates feedback systems and reinforcement to promote sustained engagement (Deterding et al., 2011). We utilized the Unity Integrated Development Environment (http://unity3d.com/), which is capable of creating applications for iOS, Android, and Web browsers. The resulting application displays engaging graphics while using few system resources. Thus, NESTT is scalable and can work on a variety of hardware platforms, including older devices that are more common within the refugee community. Unity also allows for efficient updates to the software and dissemination of these updates with minimal effort.

The language-free graphical interface allows the application to be accessed by multiple cultural groups irrespective of language of origin. The inclusion of interpreters, cultural experts, and refugees in each stage of development ensured that the final product meets the needs of clients. For example, users create an avatar visually representing themselves (e.g., skin color, hair color, hair style) that reflects cultural and/or religious dress and ornamentation (e.g., sari, hijab, bindi, facial piercings). The user is able to change the displayed emotion of the avatar to reflect his or her own emotional state while utilizing the NESTT toolkit (See Figure 1). Users are also prompted with cultural and/or religious images throughout the NESTT toolkit (e.g., puja, mala beads, temple and/or mosque safety exercise) (See Figure 2).

Figure 1. Avatar Emotion.
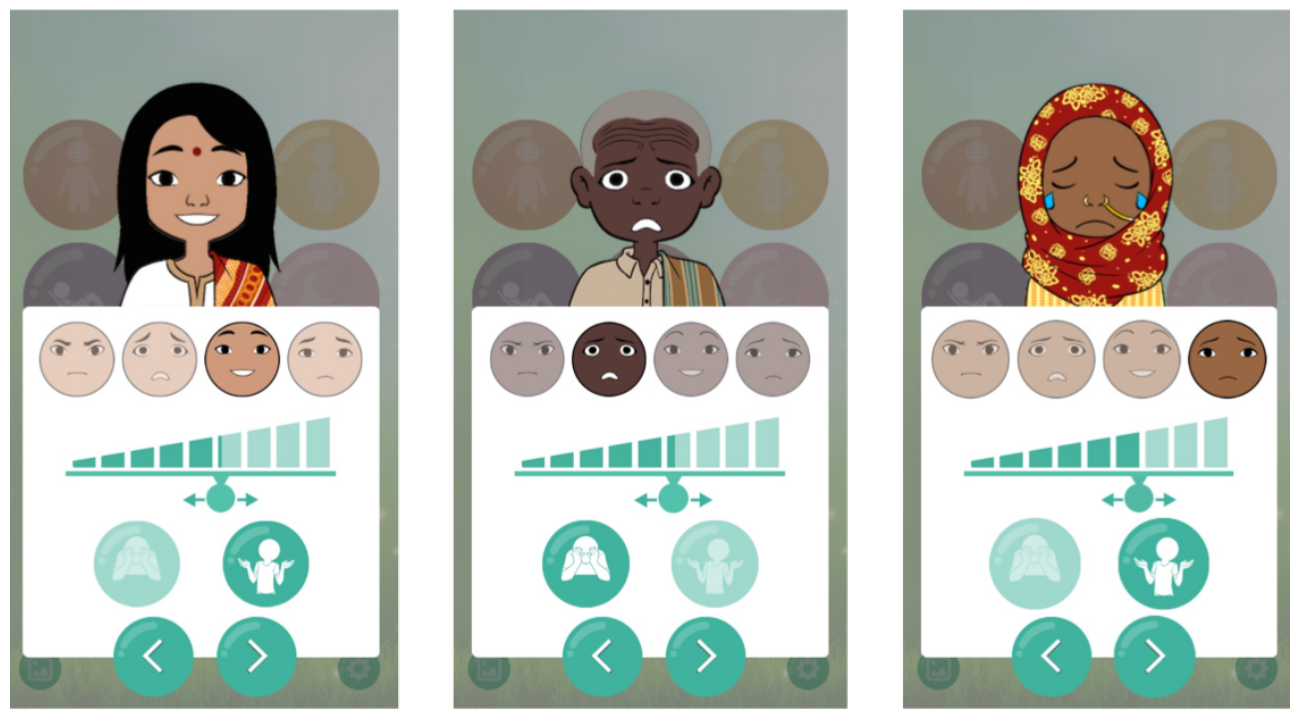

\section{Chronic Traumatic Stress (CTS) Framework and Treatment (CTS-T)}

The CTS Framework (Fondacaro Mazzulla, 2018) and CTS-T (Mazzulla Fondacaro, 2018) was developed to help guide clinical work and empirical investigation of interventions for refugee mental health. The evidence-based techniques incorporated in each module of CTS-T were used during our pilot intervention. These modules are aimed at reducing the chronic symptoms that 
Figure 2. Example of Safety Exercise.

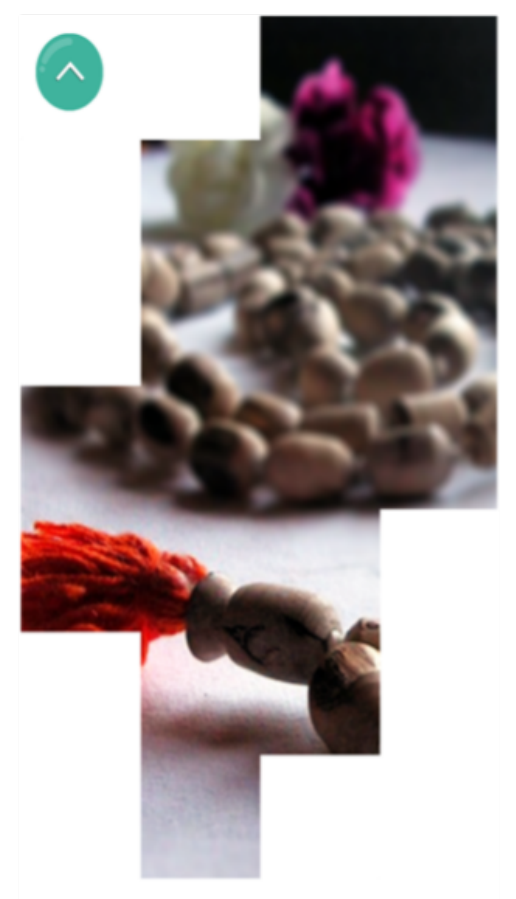

interfere with daily functioning and increasing acceptance of trauma-related emotions, coping skill use, and values-driven behavioral activation (Fondacaro Mazzulla, 2018; Mazzulla Fondacaro, 2018). The CTS-T intervention promotes skills to live a valued and fulfilling life despite the pervasiveness of the pain and suffering patients have experienced or are currently experiencing. The intervention is delivered in a weekly group format utilizing empirically supported strategies for the treatment of functional impairment associated with trauma and torture. The communal atmosphere of the group emphasizes the importance of community support and is a first step in re-accessing community as an important life value (Bunn et al., 2018). Although the full treatment includes ten modules (Mazzulla Fondacaro, 2018), a brief intervention centering on utilization of the NESTT application was designed for this pilot study (See Figure 3). Modules included a mental health discussion; safety; values; behavioral activation and exercise; coping skills and emotion regulation; sleep hygiene; acceptance and tolerance of emotions; cognitive restructuring and defusion; life-path exercise. These modules were designed specifically to address war-related traumas and torture.

\section{Measures}

Day 1 pre-treatment assessment measures included The Refugee Health Screener-15 (RHS15; Hollifield et al., 2013), the Valued Living Questionnaire (Wilson et al., 2010), and an investigator generated coping measure. These measures were also administered post-treatment to determine clinical symptoms and behavior change as a result of the mHealth intervention. The RHS-15 is a validated and widely used measure of emotional distress and mental health for refugee populations with excellent internal consistency (Chronbach's $=0.92$ ) indicating item reliability. The RHS-15 is also a valid measure for predicting cases of clinically relevant depression, anxiety, somatization, and PTSD and is highly sensitive (range .81 to .95) and specific (range .86 to .89) (Hollifield et al., 2013). The RHS-15 is a fifteen-item mental health screen assessing symptoms of depression, anxi- 
Figure 3. Overview of program elements.

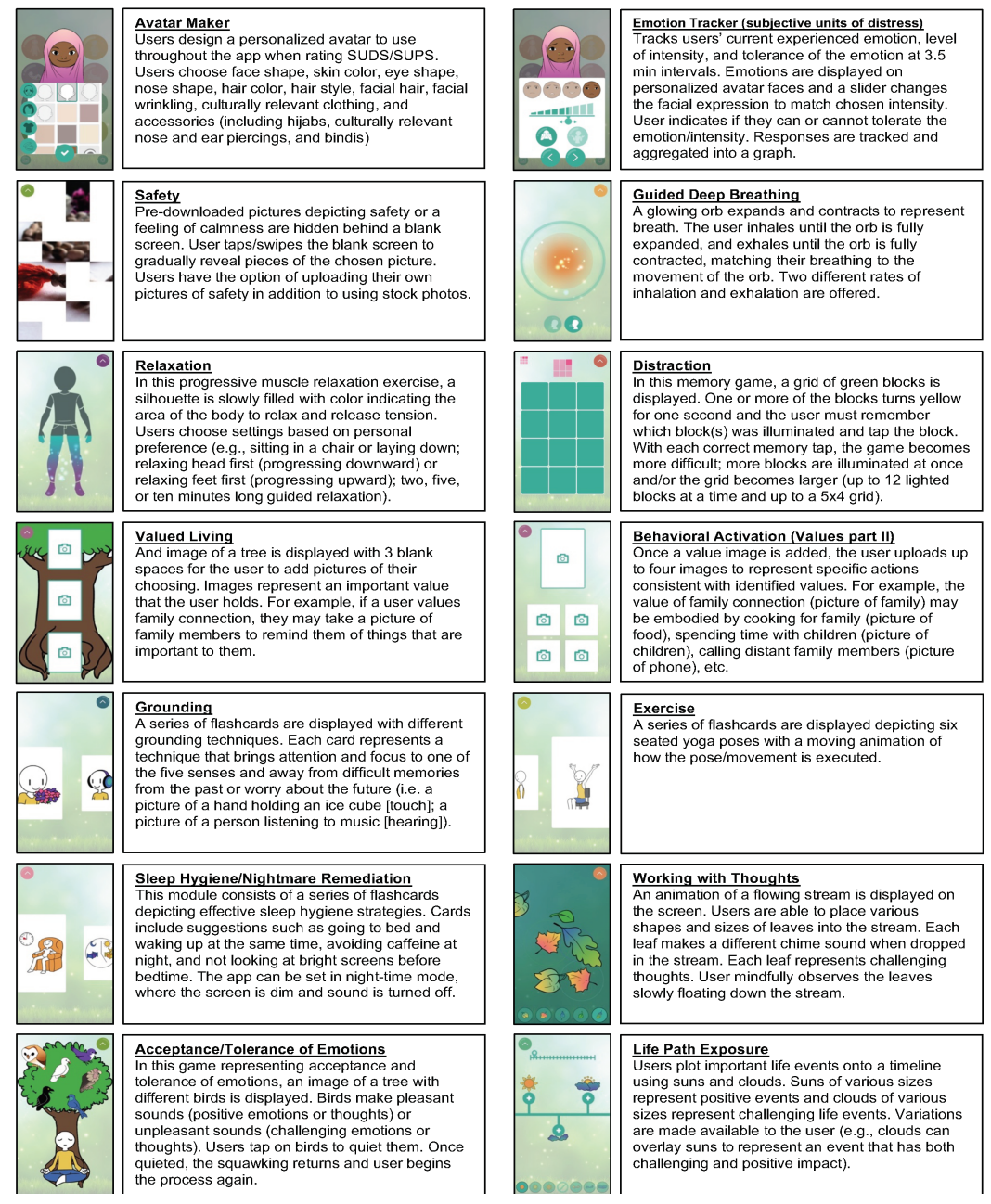

ety, somatization, and post-traumatic stress. It was developed specifically for a refugee population with the help of refugees originating from Myanmar, Bhutan, and Iraq who were recently re-settled to the United States. Items number 1-14 ask participants to rate the frequency with which psychological and somatic symptoms are experienced on a 5-point Likert scale pictorially represented with a glass filled to varying degrees and scored 0 ('not at all') to 4 ('extremely'). Item fifteen is a distress thermometer requesting participants rate experienced level of distress from 0 ('no distress') to 10 ('extreme distress'). A total score 12 on items 1-14 and/or a score of 5 on the distress thermometer are considered to be a positive screen requiring further assessment. A total score (psychosocial distress), and four sub-scores (depression, anxiety, somatization, and traumatic stress) were calculated. Higher scores are indicative of greater distress within each domain.

The Valued Living Questionnaire (VLQ) is a validated two-part questionnaire designed to assess the level to which an individual engaged in value driven behavior across 10 domains within the past week. In the first part, participants rate the importance of 10 domains of living (family; marriage/couples/intimate relations; parenting; friendship; work; education; recreation; spirituality; citizenship; physical self-care). The VLQ has good internal consistency (Chronbach's $=0.77$ ) indicating item reliability. In the second portion, participants rate, on a 10-point Likert scale $(0=$ Not at All; $10=$ Extremely), how consistent their behavior has been with identified values within each domain. A total score was calculated to represent value driven behavioral engagement with higher scores indicative of better engagement. Finally, an investigator generated coping measure that assessed 
the frequency with which participants engaged in the coping skills (e.g., relaxation, deep breathing, grounding, distraction, mindfulness) relevant to the intervention was administered. Participants responded on a four-point Likert scale $(0=$ Not at All; $3=$ A lot $)$ regarding the frequency with which he or she engaged in the identified coping skills. A total score was calculated to represent coping skill engagement in which higher scores are indicative of greater frequency of coping behaviors.

\section{Testing Protocol}

A six-week pilot test (two weeks of assessment and four weeks of intervention) was conducted in group format (10 women-group 1; 8 men-group 2) during which the CTS-T with the NESTT application was utilized. Session 1 and session 6 were individual assessment appointments, lasting 60 minutes each. During these individual meetings, the clinician-researcher administered the study battery using an interpreter. Sessions 2 through 5 included the group-based CTS-T protocol as well as review of applicable tools from the NESTT app. Each group session was 90 minutes in duration and included interpreters for each language represented. Details of each session is provided below.

Prior to the first group meeting (Study Day 1), each participant met individually with a clinician-researcher for a pre-intervention assessment lasting approximately 60 minutes. After obtaining consent for participation in the research study using an interpreter, participants were administered a brief assessment battery. Assessment measures included: The Refugee Health Screener-15 (RHS-15), the Valued Living Questionnaire, and an investigator generated coping measure (see measures).

The first group meeting (Study Day 2), lasting 90 minutes, utilized one interpreter for each language represented (Nepali; Maay Maay) and consisted of an introduction to CTS-T and an orientation to the tablet utilized in the pilot study. The introduction to CTS-T included a discussion of torture and trauma and the range of expected psychological and physiological responses to traumatic experiences (CTS-T Module 1; Mazzulla Fondacaro, 2018). The orientation to the tablet included a functional overview of the device and opportunities for practicing access of the application (e.g., turning the device on, opening and closing the NESTT application, making personalized avatar, troubleshooting). Additionally, two NESTT application components (safety distraction, and breathing orb) were taught and practiced (CTS-T Module 1; CTS-T Module 2) (Mazzulla Fondacaro, 2018). Participants were encouraged to utilize the NESTT application between group meetings.

The second group meeting (Study Day 3), lasting 90 minutes, utilized one interpreter for each language represented (Nepali; Maay Maay) and consisted of a review of the previous week's application components and troubleshooting any technical concerns experienced. New topics were presented and affiliated NESTT application components were introduced (valued living, behavioral activation, relaxation, and distraction) (CTS-T Module 3, CTS-T Module 4, CTS-T Module 5) (Mazzulla Fondacaro, 2018). Opportunities to access and practice the application components were provided. Participants were encouraged to utilize the NESTT application components between group meetings.

The third group meeting (Study Day 4), lasting 90 minutes, utilized one interpreter for each language represented (Nepali; Maay Maay) and consisted of a review of the previous weeks' application components, technical troubleshooting, and an open discussion of if and when participants found the NESTT application to be helpful. New topics were presented and affiliated NESTT application components were introduced (grounding, exercise, sleep hygiene/nightmare remediation) (CTS-T Module 5, CTS-T Module 6) (Mazzulla Fondacaro, 2018). Opportunities to access and practice the application components were provided. Participants were encouraged to utilize any of the NESTT application components between group meetings. 
The fourth group meeting (Study Day 5), lasting 90 minutes, utilized one interpreter for each language represented (Nepali; Maay Maay) and reviewed any remaining questions about learned application components, technical troubleshooting, and an open discussion of the utility and usability of the NESTT application. The final three topics were introduced and affiliated NESTT application components were introduced and practiced (working with thoughts, acceptance and tolerance of emotions, life-path exercise) (CTS-T Module 7, CTS-T Module 8; CTS-T Module 9) (Mazzulla Fondacaro, 2018). Opportunities to access and practice the application components were provided. Clinician-researchers encouraged participants to utilize any of the NESTT application components between group meetings.

The last meeting (Study Day 6) was conducted individually and lasted approximately 60 minutes. Using an individual interpreter, a clinician-researcher administered the brief assessment battery to participants. Assessment measures included The Refugee Health Screener-15 (RHS-15), the Valued Living Questionnaire, and an investigator generated coping measure (see measures).

User data were collected from participants between group meetings measuring between session engagement. The NESTT application was designed to assess engagement via software and metrics embedded in the unit. These metrics include: 1) Frequency of accessing application "tools"; 2) Duration/Time spent using the application "tools"; 3) Time of day of accessing application "tools". User inputs (screen taps) per "tool"; Bug reports; User errors. These metrics determine how the application is used, when it is used, and which components are used most extensively (See Table 2).

\section{Results}

Our results indicated a reduction in trauma related symptomology and an increase in coping skills and functioning. Paired-samples t-tests were conducted to compare pre- and postintervention levels of combined psychosocial distress, depression, anxiety, somatization, traumatic stress, coping, and valued living. Results indicated that following the completion of the NESTT intervention, valued living scores did not change, however, participants experienced a significant reduction in overall psychosocial distress $\mathrm{t}(17)=15.05, \mathrm{p}<.001$; depression $\mathrm{t}(17)=12.78, \mathrm{p}<.001$; anxiety $t(17)=9.59, p<.001$; somatic complaints $t(17)=9.31, p<.001$, traumatic stress $t(17)=12.23, p<.001$, and an increase in utilization of coping skills $t(17)=-12.55, p<.001$ (see Table 1. The effect size for these analyses were found to exceed Cohen's (1988) convention for a large effect ( $d=.80$ ) (see Table 1). Importantly, all participants had a pre-intervention total psychosocial distress score at or above 12 , which is classified as clinically significant per RHS-15 criteria. Following the six-week intervention, only three participants (16\%) continued to exhibit clinically significant distress.

Table 1. Descriptive statistics and paired samples t-test results

\begin{tabular}{rllll}
\hline Outcome & Pre-Test M (SD) & Post-Test M (SD) & t-value & Cohen's D \\
\hline Psychosocial Distress & $32.17(7.25)$ & $7.61(5.77)$ & $15.05^{* *}$ & 6.92 \\
Depression & $9.61(2.87)$ & $2.22(2.07)$ & $12.78^{* *}$ & 2.45 \\
Anxiety & $6.56(2.41)$ & $1.50(1.29)$ & $9.59^{\star *}$ & 2.23 \\
Somatization & $5.72(1.60)$ & $1.78(1.31)$ & $9.31^{* *}$ & 1.79 \\
Traumatic Stress & $10.28(2.59)$ & $2.11(1.84)$ & $12.23^{* *}$ & 2.83 \\
Coping Behaviors & $3.33(3.31)$ & $13.89(1.32)$ & $-12.55^{\star *}$ & 3.56 \\
Valued Living & $73.83(16.02)$ & $80.72(24.92)$ & -0.87 & 0.33 \\
\hline
\end{tabular}

To assess participant engagement with NESTT, we gleaned metrics from the usability software embedded in Unity including: Frequency of access; Total time spent; and Times tools were 
accessed (see Table 2). These metrics helped to determine how the application was used, when it was used, and which components were used most extensively during the pilot testing. At the end of the intervention, participant perception of the NESTT usability was assessed. Ninety-five percent of participants reported that they "somewhat" to "very much" agreed that the app was easy for them to use; $83 \%$ of participants reported that they "somewhat" to "very much" agreed that the app was clear and understandable; $100 \%$ of participants reported that the app was easy to incorporate into their daily lives. All participants reported weekly use of the NESTT. All tools within NESTT were utilized.

Table 2. NESTT Usability Data

\begin{aligned} & \hline Metric Outcome \\ & \hline Average number of times accessed per week 9.83 \\ & Average number of times accessed per day 1.39 \\ & Average number of minutes spent per day 17.3 \\ & Time of Day Accessed $6 \mathrm{am}-12 \mathrm{pm}: 12.23 \% \\ & 12 \mathrm{pm}-6 \mathrm{pm}: 70.41 \% \\ & 6 \mathrm{pm}-12 \mathrm{am}: 17.24 \% \\ & 12 \mathrm{am}-6 \mathrm{am}: 0.00 \% \\ &$\hline\end{aligned}

\section{Discussion}

Refugees often experience stressors resulting in psychological symptomology such as posttraumatic stress disorder (Johnson Thompson, 2008; Moisander Edston, 2003), depression, generalized anxiety disorder, and somatic concerns (Jaranson et al., 2004; Mollica et al., 1997). Additionally, refugees re-settled in the United States face a range of post-migration challenges such as navigating a new society, learning English, obtaining gainful employment, experiencing discrimination, and problem-solving housing challenges. Not surprisingly, past trauma and persistent stressors often result in functional impairment requiring culturally adapted intervention. Culturally adapted treatments, including Chronic Traumatic Stress Treatment (CTS-T), focus on valued living, emotion regulation, and skills to manage acute distress (Mazzulla Fondacaro, 2018; Nicholl Thompson, 2004).

The mHealth application developed by the authors is a language-free toolkit of culturally adapted, evidence-based treatment techniques for trauma related symptoms. Given the languagefree format of NESTT, refugees from diverse backgrounds can utilize this toolkit irrespective of English proficiency, addressing one of the largest disparities in mental health service provision to refugees. The NESTT mHealth application utilized in this pilot-study successfully addresses additional barriers such as limited access to affordable healthcare (this application is a free, downloadable tool) and transportation challenges (this application is compatible for use on personal devices in any location). Importantly, this tool was developed utilizing cultural consultants from two distinct re-settled cultural groups to ensure that evidence-based intervention strategies were communicated in a culturally relevant manner. The tools incorporated in our application map on to evidence-based treatment modalities utilized in culturally adapted mental health treatment for refugees.

The current study indicated that the NESTT application has the potential to improve mental health service provision for refugees. Indeed, engagement with evidence-based techniques delivered via mobile devices provides an affordable and effective way to assist refugees in need of critical mental health support. Specifically, NESTT is a tool that can supplement traditional means 
of service delivery or allow access to evidence-based intervention tools to individuals not already connected to mental health services.

For those working with a clinician, the ability to practice evidence-based interventions between in-person therapy sessions and during crisis situations has the potential for positively impacting both service provider and client. NESTT is designed to be introduced during individual or group intervention and can be utilized independently between sessions and after treatment is concluded. Access to strategies between sessions results in faster skill acquisition, shorter interventions, and maintenance of treatment gains. Literature suggests that refugees regularly use high-cost services, such as emergency departments, in response to extreme acute distress (Fernandez et al., 2004; Miller, 1995). The NESTT application may help to reduce the frequency of emergency room visits and 911 calls, by allowing individuals to access effective coping strategies during a non-medical emergency. Furthermore, the discrete nature of a mHealth toolkit used on a private mobile device such as a phone allows clients to access effective intervention resources electronically and on demand.

Data from this pilot study demonstrated the utility of this approach through a significant decrease in trauma related symptoms (anxiety, depression and somatic symptoms), and increased use of coping skills. Data collected in this pilot study indicated that mHealth treatments are a useful supplement or introduction to mental health strategies. The NESTT application improves the quality of care currently available for refugees in need of critical mental health support.

No significant differences between pre and post intervention valued living scores were detected. This may be due to the unexpected high levels of engagement in valued living activities reported during the pre-assessment session. These scores indicated that on average, participants were engaging in valued living behaviors prior to the intervention.

Limitations of the current study include a small sample size. Caution is recommended when interpreting underpowered samples to test hypotheses as to the efficacy of a treatment or calibrate effect sizes due to the inflated risk of Type I or Type II error. However, our results indicated reduction in trauma related symptomology and increase in coping skills and functioning in addition to revealing encouraging usability data. It is suggested that this paradigm be replicated in a larger sample to confirm these promising results. Additionally, the pilot study was conducted in a group setting during which orientation to evidence based application components was given. Although this was not a therapy group and didactic content was restricted to the application components, in person instruction was provided by trained therapists.

The results of the present study indicate that the use of a mHealth application improves mental health outcomes in a sample of refugees re-settled in the United States. Future research related to the current study may include an investigation of treatment groups with and without the use of the NESTT app between sessions. This type of investigation would help researchers understand the degree to which between session practice with a m-Health application improves symptoms and increases coping skills. Additionally, although there are general principles to implementing cultural adaptations to evidence-based interventions (e.g., utilizing an interpreter; learning greetings and key therapy terms in language of origin), each culture is uniquely different. The NESTT application was carefully designed with the assistance of cultural consultants to reflect norms, images, and concepts from the Somali-Bantu and Nepali-Bhutanese cultures specifically. Many aspects of the NESTT application can be utilized with other cultures, however, certain application components will not attend to differences across cultures. It would be beneficial to expand application details, such as cultural dress and religious images, to broaden the applicability of this tool. 


\section{References}

Amstadter, A. B., Broman-Fulks, J., Zinzow, H., Ruggiero, K. J., and Cercone, J. (2009). Internet-based interventions for traumatic stress-related mental health problems: a review and suggestion for future research. Clinical psychology review, 29(5):410-420.

Bakker, D., Kazantzis, N., Rickwood, D., and Rickard, N. (2016). Mental health smartphone apps: review and evidence-based recommendations for future developments. JMIR mental health, 3(1):e4984.

Bunn, M., Goesel, C., Kinet, M., and Ray, F. (2016). Group treatment for survivors of torture and severe violence: A literature review. Torture Journal, 26(1):23-23.

Bush, N. E., Skopp, N., Smolenski, D., Crumpton, R., and Fairall, J. (2013). Behavioral screening measures delivered with a smartphone app: psychometric properties and user preference. The Journal of nervous and mental disease, 201(11):991-995.

Campbell, T. A. (2007). Psychological assessment, diagnosis, and treatment of torture survivors: a review. Clinical psychology review, 27(5):628-641.

Deterding, S., Sicart, M., Nacke, L., O'Hara, K., and Dixon, D. (2011). Gamification. using game-design elements in non-gaming contexts. In CHI'11 extended abstracts on human factors in computing systems, pages 2425-2428.

Fernandez, W. G., Galea, S., Ahern, J., Sisco, S., Waldman, R. J., Koci, B., and Vlahov, D. (2004). Mental health status among ethnic albanians seeking medical care in an emergency department two years after the war in kosovo: a pilot project. Annals of emergency medicine, 43(2):E1-E8.

Foa, E. B., Chrestman, K. R., and Gilboa-Schechtman, E. (2008). Prolonged exposure therapy for adolescents with PTSD emotional processing of traumatic experiences, therapist guide. Oxford University Press.

Fondacaro, K. and Mazulla, E. (2018). The chronic traumatic stress framework: a conceptual model to guide empirical investigation and mental health treatment for refugees and survivors of torture. Torture Journal, 28(1).

Fondacaro, K. M. and Harder, V. S. (2014). Connecting cultures: A training model promoting evidence-based psychological services for refugees. Training and Education in Professional Psychology, 8(4):320.

Glenn, D., Golinelli, D., Rose, R. D., Roy-Byrne, P., Stein, M. B., Sullivan, G., Bystritksy, A., Sherbourne, C., and Craske, M. G. (2013). Who gets the most out of cognitive behavioral therapy for anxiety disorders? the role of treatment dose and patient engagement. Journal of consulting and clinical psychology, 81(4):639.

Hollifield, M., Verbillis-Kolp, S., Farmer, B., Toolson, E. C., Woldehaimanot, T., Yamazaki, J., Holland, A., Clair, J. S., and SooHoo, J. (2013). The refugee health screener-15 (rhs-15): development and validation of an instrument for anxiety, depression, and ptsd in refugees. General hospital psychiatry, 35(2):202-209.

Jaranson, J. M., Butcher, J., Halcon, L., Johnson, D. R., Robertson, C., Savik, K., Spring, M., and Westermeyer, J. (2004). Somali and oromo refugees: correlates of torture and trauma history. American journal of public health, 94(4):591-598.

Johnson, H. and Thompson, A. (2008). The development and maintenance of post-traumatic stress disorder (ptsd) in civilian adult survivors of war trauma and torture: A review. Clinical psychology review, 28(1):36-47.

Kiluk, B. D., Buck, M. B., Devore, K. A., Babuscio, T. A., Nich, C., and Carroll, K. M. (2017). Performance-based contingency management in cognitive remediation training: a pilot study. Journal of substance abuse treatment, 72:80-88.

Krebs, P. and Duncan, D. T. (2015). Health app use among us mobile phone owners: a national survey. JMIR mHealth and uHealth, 3(4):e4924.

Kuhn, E., Greene, C., Hoffman, J., Nguyen, T., Wald, L., Schmidt, J., Ramsey, K. M., and Ruzek, J. (2014). Preliminary evaluation of ptsd coach, a smartphone app for post-traumatic stress symptoms. Military medicine, 179(1):1218.

Luxton, D. D., McCann, R. A., Bush, N. E., Mishkind, M. C., and Reger, G. M. (2011). mhealth for mental health: Integrating smartphone technology in behavioral healthcare. Professional Psychology: Research and Practice, 42(6):505. 
Mazzulla, E., F. K. M. (2018). Chronic traumatic stress treatment (cts-t); a mental health intervention designed for refugees and survivors of torture. https://www. newenglandsurvivorsoftorture.org/cts-t-manual.

Miller, J. A. (1995). Caring for cambodian refugees in the emergency department. Journal of Emergency Nursing, 21(6):498-502.

Minkler, M. and Wallerstein, N. (2011). Community-based participatory research for health: From process to outcomes. John Wiley \& Sons.

Moisander, P. A. and Edston, E. (2003). Torture and its sequel-a comparison between victims from six countries. Forensic science international, 137(2-3):133-140.

Mollica, R. F., Poole, C., Son, L., Murray, C. C., and Tor, S. (1997). Effects of war trauma on cambodian refugee adolescents' functional health and mental health status. Journal of the American Academy of Child \& Adolescent Psychiatry, 36(8):1098-1106.

Nicholl, C. and Thompson, A. (2004). The psychological treatment of post traumatic stress disorder (ptsd) in adult refugees: A review of the current state of psychological therapies. Journal of Mental Health, 13(4):351362.

Price, M., Williamson, D., McCandless, R., Mueller, M., Gregoski, M., Brunner-Jackson, B., Treiber, E., Davidson, L., and Treiber, F. (2013). Hispanic migrant farm workers' attitudes toward mobile phone-based telehealth for management of chronic health conditions. Journal of medical Internet research, 15(4):e76.

Price, M., Yuen, E. K., Goetter, E. M., Herbert, J. D., Forman, E. M., Acierno, R., and Ruggiero, K. J. (2014). mhealth: a mechanism to deliver more accessible, more effective mental health care. Clinical psychology \& psychotherapy, 21(5):427-436.

Resick, P. A. and Schnicke, M. (1993). Cognitive processing therapy for rape victims: A treatment manual, volume 4. Sage.

Rose, G. L., Skelly, J. M., Badger, G. J., Ferraro, T. A., and Helzer, J. E. (2015). Efficacy of automated telephone continuing care following outpatient therapy for alcohol dependence. Addictive behaviors, 41:223-231.

Ruzek, J., Hoffman, J., Ciulla, R., Prins, A., Kuhn, E., and Gahm, G. (2011). Bringing internet-based education and intervention into mental health practice: Afterdeployment. org. European Journal of Psychotraumatology, 2(1):7278.

Stecker, T., McHugo, G., Xie, H., Whyman, K., and Jones, M. (2014). Rct of a brief phone-based cbt intervention to improve ptsd treatment utilization by returning service members. Psychiatric Services, 65(10):1232-1237.

Wilson, K. G., Sandoz, E. K., Kitchens, J., and Roberts, M. (2010). The valued living questionnaire: Defining and measuring valued action within a behavioral framework. The Psychological Record, 60(2):249-272. 